\title{
Turbulent Solution of the Navier-Stokes Equations
}

R. G. Deissler

Lewis Research Center

Cleveland, Ohio

Prepared for the

Thirty-third Annual Meeting of the Division of Fluid Dynamics of the American Physical Society Ithaca, New York, November 23-25, 1980

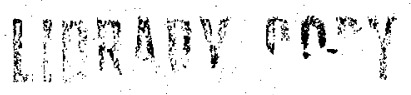

APR $1 ? 989$ 


\section{TURBULENT SOLUTION OF THE NAVIER-STOKES EQUATIONS \\ by R. G. Deisster}

\section{National Aeronautics and Space Administration \\ Lewis Research Center \\ Cleveland, Ohio 44135}

To study the nonlinear physics of incompressible turbulent flow, the unaveraged Navier-Stokes equations are solved numerically. Initial threedimensional cosine velocity fluctuations and periodic boundary conditions are used. No mean gradients are present. The three components of the mearisquare velocity fluctuations are equal for the initial conditions chosen. The resulting solution shows characteristics of turbulence, such as the nonlinear excitation of small-scale fluctuations. For the higher Reynolds numbers the initially nonrandom flow develops into an apparently random turbulence. 


\section{INTRODUCTION}

Although much turbulence research has been done in recent years, there does not appear to be a general deductive theory of high Reynolds number turbulence. Most of the analytical theories depend on a closure assumption for the hierarchy of averaged equations. This immediately calls into question the appropriateness of referring to them as deductive theories, except at low Reynolds numbers where nonlinear effects are small. ${ }^{1}$

One way in which a closure assumption can be avoided is by closure by specification of initial conditions. 2 That method can successfully predict turbulent decay. However, in order to use it the initial conditions must be fully turbulent, and a large amount of initial data is required to satisfactorily specify the initial turbulence. Moreover, the method appears limited to decaying turbulence and does not seem capable of extension to turbulence maintained by mean gradients, where the effect of initial conditions eventually becomes negligible.

In view of the foregoing comments it seems desirable to consider a numerical solution of the Navier-Stokes equations which displays features of turbulence. It might be pointed out that it is more appropriate to refer to a numerical solution of the unaveraged equations as deductive than it is to so refer to most of the analytical theories, where closure assumptions are required. Several numerical solutions of the unaveraged equations have appeared which use a spectrum of random initial conditions (e.g., Refs. 3 and 4). These studies appear to demonstrate the feasibility of carrying out turbulent solutions with present-day computing equipment and certainly represent major advances.

Because of the difficulty of specifying realistic turbulent initial conditions (experimentally or analytically), it may be more appropriate to 
initially specify a simple regular fluctuation (as actually occurs downstream of a grid). This should be better for studying the development of smallscale fiuctuations than would a spectrum of initial fluctuations, since for the latter, small-scale fluctuations are already present in the initial flow. Taylor and Green ${ }^{5}$ and others ${ }^{6,7}$ have used a perturbation series to calculate the nonlinear development of higher harmonics from lower ones. However, those studies were limited to short times or low Reynolds numbers. Orszag and Fateman ${ }^{8}$ have recently used Taylor and Green's initial conditions and obtained a numerical solution for higher Reynolds numbers and larger times. The inviscid (infinite Reynolds number) case was investigated in some detail by Betchov and Szewczyk. ${ }^{9}$

In the present paper we study the nontinear physics of turbulence numerical1y. The initial conditions are chosen to give a flow with turbulentlike properties. Unlike the problem of Taylor and Green, all three of the directional components of the mean-square velocity fluctuations are equa1. This is a simpler type of flow than that considered previously, in that it is only necessary to follow the evolution of one velocity component. We are assured that if one component has the desired turbulent or turbulentlike characteristics, the overall flow will have them. That is not the case for all initial conditions. For instance initial conditions tried earlier gave two mean-square velocity components which were equal and decayed rapidly like turbulence, but the third decayed much more slowiy. One of the objectives is to study the nonlinear excitation of smallscale fluctuations (starting with only large-scale ones) for a range of Reynolds numbers. This will be done partly by calculating the evolution of the microscale of the fluctuations. It will be seen that although the initial conditions are nonrandom, the flow at nigher Reyriolds numbers breaks up into an apparently random turbulence. 


\section{EQUATIONS AND METHODS}

The Navier-Stokes and continuity equations for an incompressible fluid can be written in dimensionless form as

and

$$
\frac{\partial u_{i}}{\partial t}=-\frac{\partial\left(u_{i} u_{k}\right)}{\partial x_{k}}-\frac{\partial p}{\partial x_{i}}+\frac{\partial^{2} u_{i}}{\partial x_{k} \partial x_{k}}
$$

$$
\frac{\partial u_{k}}{\partial x_{k}}=0
$$

where

$$
u_{i}=\frac{x_{0}}{v} u_{i}^{*}, \quad p=\frac{x_{0}^{2}}{\rho v^{2}} p^{*}, \quad t=\frac{\nu}{x_{0}^{2}} t^{*} \text {, and } x_{i}=\frac{x_{i}^{*}}{x_{0}} \text {. }
$$

Note that the stars on dimensional quantities are omitted for corresponding dimensionless quantities. The subscripts can take on the values 1, 2, and 3 , and a repeated subscript in a term indicates a summation. The quantity $u_{i}^{*}$ is an instantaneous velocity component, $x_{i}^{*}$ is a space coordinate, $x_{0}$ is a characteristic length, $t^{\star}$ is the time, $\rho$ is the density, $v$ is the kinematic viscosity, and $p^{*}$ is the instantaneous pressure. In order to obtain an explicit equation for the pressure, we take the divergence of Eq. (1) and apply the continuity Eq. (2) to get

$$
\frac{\partial^{2} p}{\partial x_{\ell} \partial x_{\ell}}=-\frac{\partial^{2}\left(u_{\ell} u_{k}\right)}{\partial x_{\ell} \partial x_{k}}
$$

In the remainder of the analysis it will be found convenient to use the set of Eqs. (1) and (3), rather than Eqs. (1) and (2).

The expression assumed for the initial disturbance is, in dimensionless form

$$
u_{i}=a_{i} \cos \vec{q} \cdot \vec{x}+b_{i} \cos \vec{r} \cdot \vec{x}+c_{i} \cos \vec{s} \cdot \vec{x},
$$


where

$$
a_{i}=\frac{x_{0}}{v} a_{i}^{*}, \quad b_{i}=\frac{x_{0}}{v} b_{i}^{*}, \quad c_{i}=\frac{x_{0}}{v} c_{i}^{*}, \quad \vec{q}=x_{0} \vec{q}^{*}, \quad \vec{r}=x_{0} \vec{r}^{*}, \quad \vec{s}=x_{0} \vec{s}^{*} .
$$

The quantities $a_{i}^{*}, b_{i}^{*}$, and $c_{i}^{*}$ are initial velocity amplitudes or Fourier coefficients of the disturbance, and $\vec{q}^{*}, \vec{r}^{*}$, and $\vec{s}^{*}$ are initial wave number vectors. The quantities $\vec{q} \cdot \vec{x}$, etc. are dot products, so that $\vec{q} \cdot \vec{x}=q_{1} x_{1}+q_{2} x_{2}+q_{3} x_{3}$, etc. In order to satisfy the continuity condition, Eq. (2),

$$
a_{i} q_{i}=b_{i} r_{j}=c_{i} s_{i}=0 \text {. }
$$

For the present work we set

$$
\begin{gathered}
a_{i}=k(2,1,1), \quad b_{i}=k(1,2,1), \quad c_{i}=k(1,1,2), \\
q_{i}=(-1,1,1), \quad r_{i}=(1,-1,1), \quad \text { and } \quad s_{i}=(1,1,-1),
\end{gathered}
$$

where $k$ is a quantity that fixes the Reynolds number. In addition to satisfying continuity, Eqs. (6) give

$$
\overline{u_{1}^{2}}=\overline{u_{2}^{2}}=\overline{u_{3}^{2}}=\overline{u^{2}}
$$

at all times, where the overbars indicate values averaged over space. Thus, Eqs. (4) and (6) give a particularly simple type of flow, in that we need specify only one component of the mean-square-velocity.

In order to carry out the numerical solution of Eqs. (1) and (3) subject to initial conditions, Eqs. (4) and (6), we use a cubical grid with faces at $x_{i}=0$ and $2 \pi$. For boundary conditions we set the values of $u_{i}$ equal at corresponding positions on opposite faces (and in the vicinity of those faces, as required for calculating numerical derivatives at the faces). That is, we assume that the boundary conditions are periodic. 
The spacial-and time-differencing schemes are essentially those used by Clark, et a1.4 That is, for the spatial derivatives in Eqs. (1) and (3) we use centered fourth-order difference expressions. 10 For timedifferencing we use a predictor-corrector method with a second-order (leapfrog) predictor and a third-order (Adams-Moulton) corrector. 11 The Poisson equation for the pressure (Eq. (3)) is solved directly (no iteration) by a fast Fourier-transform method. This method of solution was found to preserve continuity $(\nabla \cdot \vec{u} \approx 0)$ except near the ends of some of the runs, where the solutions began to deteriorate. (Another indication of incipient solution deterioration near the ends of some of the runs was that Eqs. (7) were no longer accurately satified.)

Some of the results will be extrapolated to zero mesh size in an effort to obtain more accuracy. An expression for a corrected value of the mean square velocity fluctuation $\overline{u_{c}^{2}}$ in terms of the uncorrected value $\overline{u_{u}^{2}}$, which is consistent with fourth-order spacial differencing, is

$$
\overline{u_{c}^{2}}=\overline{u_{u}^{2}}+B(t) \Delta x^{4}+C(t) \Delta x^{6}+\ldots .,
$$

where $\Delta x$ is the distance between gria points. In a plot of $\overline{u^{2}}$ against time $t$ it may be desirable to correct the time for a given $\overline{u^{2}}$ rather than $\overline{u^{2}}$ for a given time. For that purpose we have, instead of $\mathrm{Eq}$. (8)

$$
t_{c}=t_{u}+B\left(\overline{u^{2}}\right) \Delta x^{4}+c\left(\overline{u^{2}}\right) \Delta x^{6}+\ldots \cdot
$$

In choosing between Eqs. (8) and (9) we use the equation that gives the smaller correction. Both expressions are accurate for an infinite number of terms, but for a finite number of terms the expressisn that gives the smaller correction should be more nearly converged in the sense that additional terms would not significantly change the relation between $\overline{u^{2}}$ and $t$. 
The unknown values in Eq. (8) or (9) are obtained by calculating $\overline{u^{2}}$ as a function of $t$ for several different grid-point spacings $\Delta x$. For instance by truncating Eq. (9) after terms of order 6 , we calculate $t_{u}$ against $\overline{u^{2}}$ for three values of $\Delta x$ to obtain $t_{c}, B\left(\overline{u^{2}}\right)$ and $c\left(\overline{u^{2}}\right)$ for each $\overline{u^{2}}$. If the three values of $\Delta x$ differ successively by factors of two,

$$
t_{c}=\frac{1024}{945} t_{1}-\frac{16}{189} t_{2}+\frac{1}{945} t_{3}
$$

where $t_{1}, t_{2}$, and $t_{3}$ are calculated values of $t$ for three values of $\Delta x$ at a fixed $\overline{u^{2}}$. If only two $\Delta x^{\prime} s$ are used,

$$
t_{c}=\frac{16}{15} t_{1}-\frac{1}{15} t_{2}
$$

Similar equations are obtained for $\overline{u_{c}^{2}}$ at a given time.

\section{RESULTS AND DISCUSSION}

Figure 1 shows the calculated evolution of velocity fluctuations at two fixed points in space for a high Reynolds number (no correction for discretization error). In spite of the nonrandom initial conditions (Eqs. (4) and (6)) the velocity fluctuations have the appearance of those for a random turbulence. The dashed curves of $u_{1} / \bar{u}_{0}^{1 / 2}$ are for initial conditions perturbed $\sim 0.1$ percent. The perturbed curves follow the unperturbed ones for a short time and then depart sharply. Thus a very small perturbation of initial conditions causes a large change in the values of $u_{j}$ (except near $t=0$ ). On the other hand, the root-mean-square values of the velocities decrease smoothly and are unaffected by the perturbation of initial conditions. All of these features are characteristic of turbulence. Another characteristic of turbulence is that the correlation between velocities at two times should go to zero as the separation of the times increases. Figure 2 shows that this occurs for the present high Reynolds 
number calculations. For true turbulence the correlation should probably decrease smoothly with time. Figure 2(b) shows that this is nearly the case for the larger $t_{0}$ (the time that is fixed as the other time increases). At early times there is probably some nonrandom structure in the flow caused by the nonrandom initial conditions (Fig. $2(\mathrm{a})$ ).

As a further indication that the high Reynolds number flow breaks up into turbulence, we calculate the evolution of the cross correlation $\overline{u_{1} u_{2}}$. Although $\overline{u_{1}^{2}}=\overline{u_{2}^{2}}=\overline{u_{3}^{2}}$ at all times, the initial $\overline{u_{1} u_{2}}$ given by Eqs. (4) and (6) is not zero. However Fig. 3 shows that because of the apparent randomization of the flow $\overline{u_{1} u_{2}}$ goes to zero as time increases. The fluctuations in the curve at early times (as also in the curve of Fig. 2(a)), are probably caused by nonrandom structure in the flow at early times. Figures 1 to 3 show that at later times we appear to get a reasonable approximation to isotropic turbulence, although the initial conditions are nonrandom. Calculated values of velocity-derivative skewness factor for a high Reynolds number (Fig. 4) also appear to be comparable to those for isotropic turbulence. The present calculations differ from earlier ones, where turbulence was obtained by using random initial conditions. our calculations at lower Reynolds numbers give results that are less turbulentlike. Thus the fluctuations develop apparent randomness only at the higher Reynolds numbers.

A question remains as to the source of the randomness observed in Fig. 1. Until recently it was generally assumed that randomness in a turbulent flow is due to randomness in the initial conditions, to random external fluctuations, and/or to the presence of so many eddies or harmonic components (or of so many degrees of freedom) that the identity of the individual eddies is lost. 12,13 In the present results the first two of these 
have already been eliminated, thus leaving only the proliferation of eddies or harmonic components as a source of apparent randomness. 6 However Lorenz and others ${ }^{12-15}$ nave shown that a system of nonlinear equations with only a few degrees of freedom can develop an apparently random behavior in time as a result of loss of stability of the solutions. This happens because of the cccurrence of so-called strange attractors, which are regions in the phase space of the system to which solutions are attracted. Randomness arises in those regions from a haphazaro movement of the phase point among the neighborhoods of various critical points in the phase space (points of unstable equiliorium where the solution is steaay state). Strange attractors can occur even with a small number of degrees of freedom (two or three).

The system of spacially-differenced equations used here (disregard time differencing), which is obtained from the Navier-Stokes equations, consists of ordinary differential equations of essentially the sarne form as those in the Lorenz system. ${ }^{14,15}$ In particular, both systems have nonlinear algebraic terms that contain products of two dependent variables. Since a strange attractor arising from those nonlinear terms is known to produce randomization in the Lorenz system, it appears that such attractors should also produce randomization in the differenced Navier-Stokes system. For a sufficiently fine grid there should be no significant difference between results from the und ifferenced and differenced versions of the Navier-Stokes equations, so that it seems reasonable to assume that strange attractors play a role in Navier-Stokes turbulence. The same conclusion is reached if the spectral rather than the differenced form of the Navier-Stokes equations is used, ${ }^{8}$ since those equations also have the same form as the Lorenz equations. 
In an actual turbulent flow, as in the model used here, there is a very large number of eddies or degrees of freedom, so that although strange attractors probably produce randomization, additional apparent randomization will almost certainly be produced by proliferation of eadies or harmonic components. It appears that no conclusions can be arawn as to the relative importance of the two processes. The sensitivity of the flow to initial conditions and the suddenness with which the effect of perturbation of initial conditions becomes manifest (Fig. 1) are results often associated with strange attractors. ${ }^{12-15}$ on the other hand those results could be caused in large measure by the nonlinear production of harmonic components, since, particularly at higher Reynolds numbers, the latter tends to be explosive (each harmonic component interacts with every other one). 6

It might be thought that larger Reynolds numbers would favor randomization by proliferation of harmonic components, since that process is stronger at high than at low Reynolds numbers. However the larger number of ciegrees of freedom at high Reynolds numbers will correspond to a greater number of unstable equilibrium points in the phase space of the system (where the solution is steady state). There will then be more opportunities for randomization in strange attractors. Thus at high Reynolds numbers there are arguments for randomization both by proliferation of harmonic components and in strange attractors, and both will probably occur.

The presence of strange attractors may be fortunate from a numerical standpoint, in that it should enable turbulent solutions that are qualitatively correct (at least insofar as they appear ranciom in time) to be obtained with a relatively coarse grid. A calculation which used a grid with only $3^{3}$ points gave results which appeared to be about as random as those in Fig. 1, where $32^{3}$ points were used. The use of a fine threedimensional grid, of course, requires a large amount of computer time. 
In the results given so far, no correction for the discretization error due to the finite numerical mesn size was applied. The primary purpose of the present work is to stuay the physics of turbulence rather than to obtain highly accurate results (possibly unattainable at very high Reynolds numbers). For low Reynolas numbers Fig. 5 shows that surprisingly good results can be obtained even with coarse grids. At higher Reynolds numbers the results, although less accurate, should still be qualitatively correct. Their accuracy can be improved by extrapolating to zero mesh size by applying fourth-order discretization corrections as explained in the last section. This is done in lieu of subgrid modeling, which has been used in previous work. 4 The method is related to subgrid modeling in that it assumes that the subgrid eddies are somewhat similar to the calculated eddies, but does not require the introduction of a subgrid eddy viscosity. In all of the remaining results the fourth-order discretization corrections are applied. However the corrections are negligibly small except at the nighest Reynolds number. (An alternative (possibly more accurate) method of extrapolating to zero mesh size would be to perform the extrapolation at each time step (or for a group of steps) as the numerical calculation proceeds, rather than after all the calculations for the various mesh sizes are completed. However that procedure would require a special program and was not used here.)

Figure 6 shows the calculated evolution of mean-square velocity fluctuations for a series of initial Reynolds numbers. As the Reynolds number increases ( $\nu$ and $x_{0}$ held constant), the rate of decay of $\overline{u^{2}}$ irmcreases sharply, as in experimental turbulent flows. 2 This can be attributed to the nonlinear excitation of smal1-scale turbulentiike fluctuations at the nigher Reyrolds numbers. The high shear stresses between the small eddies cause a rapid decay. 
The development of the small-scale eddies is seen more clearly in Fig. 7 , where microscale $\lambda$ calculated from

$$
\lambda^{2}=-\frac{10 u u^{\star 2}}{d u^{\star 2} / d t^{*}}
$$

(Ref. 16) is plotted against dimensionless time. As the Reynolds number increases, the small-scale structure becomes finer. The microscale decreases until the fluctuation level (inertial effect) is low enough that viscous forces prevent further decrease. After $\lambda$ decreases to a minimum, it begins to grow. This increase of $\lambda$ with time is due to the selective annihilation of eddies by viscosity, the small eddies being the first to decay. Thus at large times only the big eddies remain. It is this period of increasing $\lambda$ that is generally observed experimentally in gridgenerated turbulence. 16

The early period, in which $\lambda$ decreases with time, is of interest as illustrative of inter-wavenumber energy transfer. It is often pointed out 1,16 that turbulent energy is characteristically transferred from big eddies to small ones. This is associated with the nonlinear terms in the Navier-Stokes equations and can be thought of as due to a breakup of big eddies into smaller ones, or as a stretching of vortex filaments to smaller diameters. In spite of this energy transfer to smaller eddies, the experimental results generally show a growth of scale. ${ }^{16}$ This is because those results are usually for the later period showri in Fig. 7 where, although energy is transferred to smaller eddies, the arnihilation of small eddies by viscous action eventually wins out. The early period shown in Fig. 7 is of particular interest, in that the nonlinear transfer effects are truly dominant there; a sharp decrease in scale actually occurs as energy is transferred to smatler eddies. 
Mean-square vorticity or dissiptation fluctuations are plotted in Fig. 8, as calculated from Eq. (b.2.y) in Ref. 16. Although the curve for zero Reynolds number, where nonlinear effects are absent, decreases monotonically to zero, the curves for higher Reynolds numbers increase sharply for a while and then decrease. Thus the nonlinear terms in the NavierStokes equations are very effective vorticity generators and greatiy enhance the dissipation at small aric moderate times. For large times they appear to have the opposite effect, evidently because the turoulence itself decays rapidly to zero.

Figure 9 shows mean-square pressure fluctuations plotted against dimensionless time. The enhancement of the pressure fluctuations, although not as great as that of the vorticity and dissipation again appears to be due to nonlinear effects. In this case the nonlinear terms on the right side of the Poissor equation for the pressure provably have the greater effect.

Three measures of the relative importance of inertial (nonlinear) and viscous effects are shown for a moderately high initial Reynolds number in Fig. 10. The microscale Reynolds number $R_{\lambda}$, the ratio of nontinear velocity term to viscous term, and the ratio of pressure to viscous term in Eq. (1) are plotted against dimensionless time. The terms are root-meansquare values. All of those measures show a variation from a rather inertial to a weak fluctuating flow. For instance $R_{\lambda}$ varies from about yo to 0.7 . This is a much greater variation than has been obtained experimentally for a single run. The curves for the term ratios lie somewhat below that for $R_{\lambda}$. They indicate that except at early times the nonlinear inertial effects associated with velocity and with pressure do not differ greatly. 
CONCLUUING REMARKS

The results show that, at least at higher Reynolds numbers, an apparently random turbulence can develop from nonrandom initial conditions. This is indicated by the appearance of the instantaneous velocity fluctuations and by the sensitivity of trose fluctuations (and the insensitivity of average values) to small perturbations in the instantaneous initial conditions. Moreover, the two-time velocity correlation becomes small as the time between the occurrence of the two velocities increases. In addition the correlation between two componerits of the velocity becomes small as the time increases, as a result of the randomization. This correlation is not small initially, even though the three components of the mean square velocity fluctuation are equal at early as well as at late times for the initial conditions chosen. Also the calculated velocity-derivative skewness factors appear to be comparable to those for isotropic turbulence. Thus except in the initial period we evidently obtain, at the higher Reynolds numbers, a reasonably good approximation to isotropic turbulence.

The source of the observed randomness may lie in the presence of strange attractors 12 in the phase space of the system, as well as in the occurrence of a very large number of eddies or harmonic components (1arge number of degrees of freedom). However it appears that no conclusions can be drawn as to the relative importance of the two processes. (A strange attractor is a region in the phase space of the system to which solutions are attracted and in which randomness arises from a haphazard movement of the phase point among the neigborhoods of critical points (points of unstable equilibrium where the solution is steady state). It can occur even with a small number of degrees of freedom.) 
At early times the nonlinear transfer of energy from big eddies to small ones is almost completely dominant and causes a sharp decrease in the size of the microscale. This has not been generally observed experimentally or analytically because the period usuaily studied is for later times, where the anihilation of small eddies by viscous action causes the scale to grow, even though energy is being transferred to smaller eddies. This later period of scale growth is also observed in the present results.

The nonlinear terms in the equations of motion are very effective vorticity generators and increase the dissipation and the rate of decay. The increased rate of decay is related to the nonlinear transfer of energy to smaller eddies; the small eddies decay faster than the big ones because of the nigher shear stresses between the small eddies. Calculation of terms in the equations of motion shows, as might be expected, that the flow is dominated by nonlinear inertial effects at early times and by viscous effects at Tater times.

The accuracy of the numerical results can be improved somewhat by extrapolating to zero mesh size. This is done by applying a fourth-order correction for discretization which is consistent with the fourth-order spacial differencing used in the calculations. This procedure is used in lieu of subgrid modeling and does not require the introduction of a subgrid eddy viscosity.

\section{Ack now ledgement}

I should like to acknowledge the considerable work of Frank Molls in carrying out the programming and numerical computations for the results given in the paper. 


\section{REFERENCES}

1. R. G. Deissler, in Handbook of Turbulence, edited by W. Frost and T. H. Moulden (Plenum, New York, 1977), Vol. 1, p. 165.

2. R. G. Deissler, Phys. Fluids 22, 1852 (1979).

3. S. A. Orszag and G. S. Patterson, Jr., Phys. Rev. Lett. 28, 76 (1972).

4. R. A. Clark, J. H. Ferziger, and W. C. Reynolds, J. Fluid Mech. y1, 1 (1979).

5. G. I. Taylor and A. E. Green, Proc. R. Soc. London, Sec. A 158, 499 (1937).

6. R. G. Deissler, Appl. Sci., Res. 21, 393 (1970).

7. M. Van Dyke, SIAM J. App 1. Math. 28, 720 (1975).

8. S. A. Orszag, in Fluid Dynamics, edited by R. Balian and J.-L. Peube (Gordon and Breach, New York, 1977), pp. 273, 261.

9. R. Betchov and A. A. Szewczyk, Phys. Fluids 21, 871 (1978).

10. J. M. McCormick and M. G. Salvadore, Numerical Methods in Fortran (Prentice-Ha11, Englewood Cliffs, N.J., 1964), p. 38.

11. F. Ceschino and J. Kuntzmann, Numerical Solution of Initial Value Problems (Prentice-Hall, Englewood Cliffs, N.J., 1966), p. 141, example 2, and p. 143.

12. A. S. Monin, Sov., Pnys. Usp. 21, 429 (1978).

13. M. I. Rabinovich, Sov. Phys. Usp. 21, 443 (1978).

14. E. N. Lorenz, J. Atmos. Sci. 20, 130 (1963).

15. D. Ruelle, in Turbulence and Navier-Stokes Equations, Edited by R. Temam (Springer-Verlag, New York, 1976), p. 146.

16. G. K. Batchelor, The Theory of Homogeneous Turbulence (Cambridge University Press, New York, 1953), pp. 100, 137, 88, 86. 


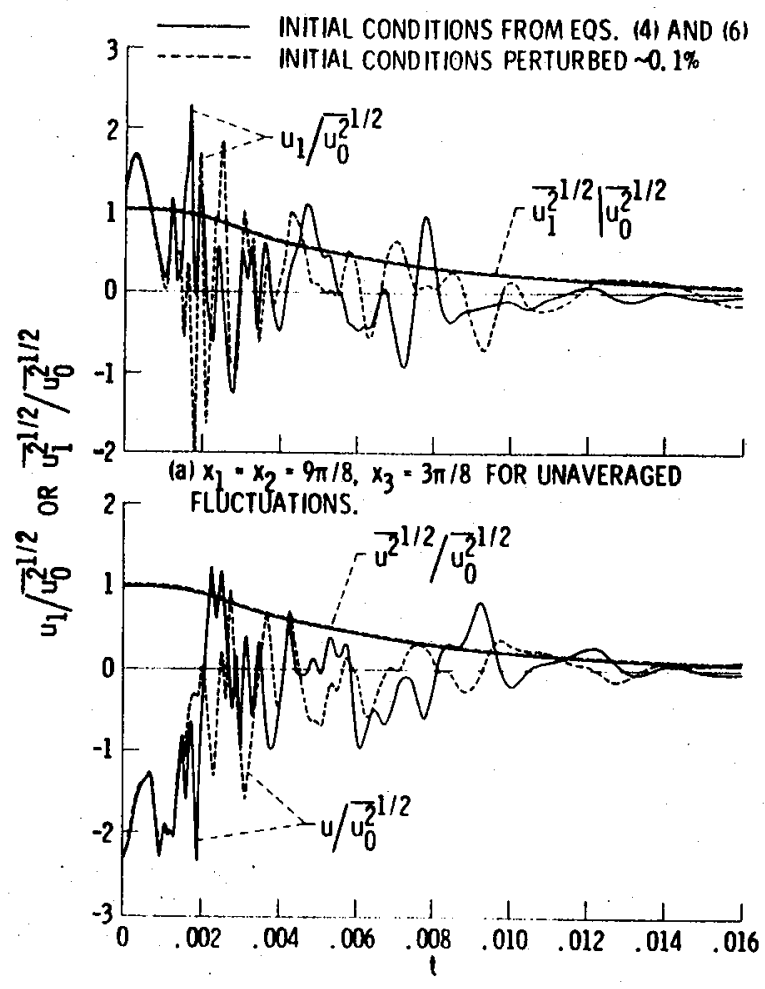

(b) $x_{1}=x_{2}=x_{3}=\pi$ FOR UNAVERAGED FLUCTUATIONS.

Fig. 1 Calculated evolution of lurbulent velocity fluctuations (normalized by initjal condition) for a high Reynolds number $u_{0}^{* 2} x_{0} / v=2217$. Root-meansquare fluctuations are spatially averaged. $\Delta x_{i}=\pi / 16$. 


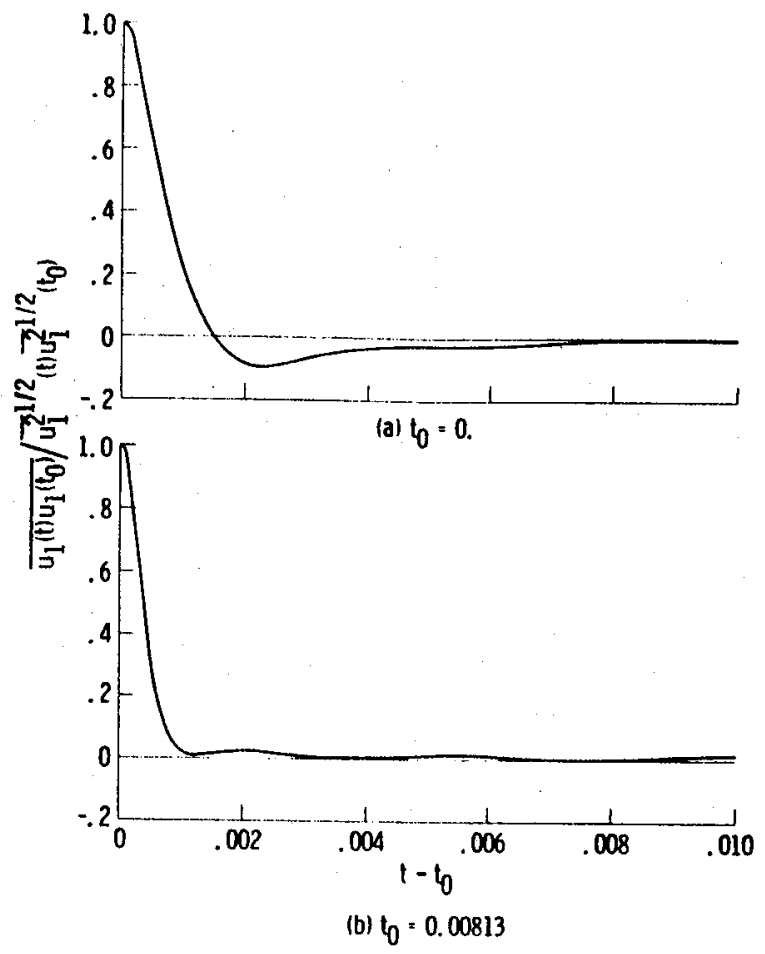

Fig. 2 Calculated correlation coefficient for velocities at dimensionless times $t$ and $t_{0}$ plotted against $t-t_{0}$. $u_{0}^{k 2 / 2} x_{0} / \nu=2217, \Delta x_{i}=\pi / 16$.

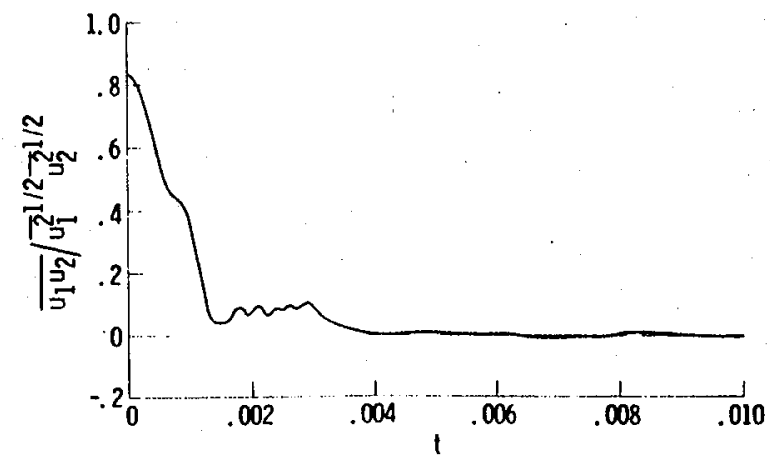

Fig. 3 Calculated correlation coefficient for two velocity components plotted against dimensionless time.

$\Delta x_{i}=\pi / 16$. 


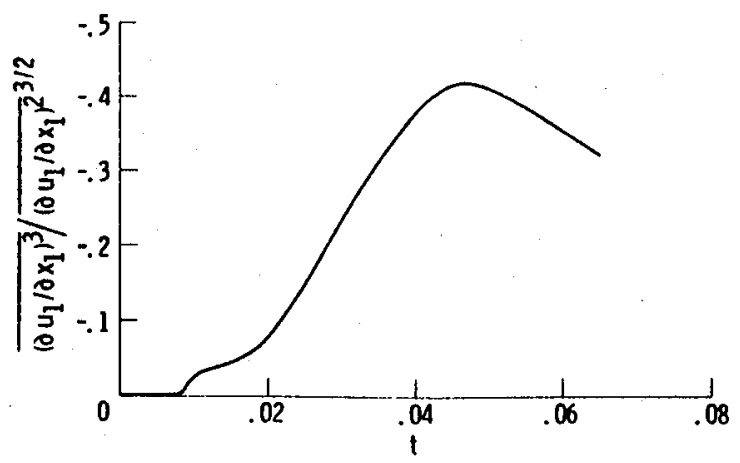

Fig. 4 Calculated evolution of velocity-derivative skewness factor. $\overline{u_{0}^{* 2}} 1 / 2 x_{0} / v=2217, \Delta x_{i}=\pi / 16$.

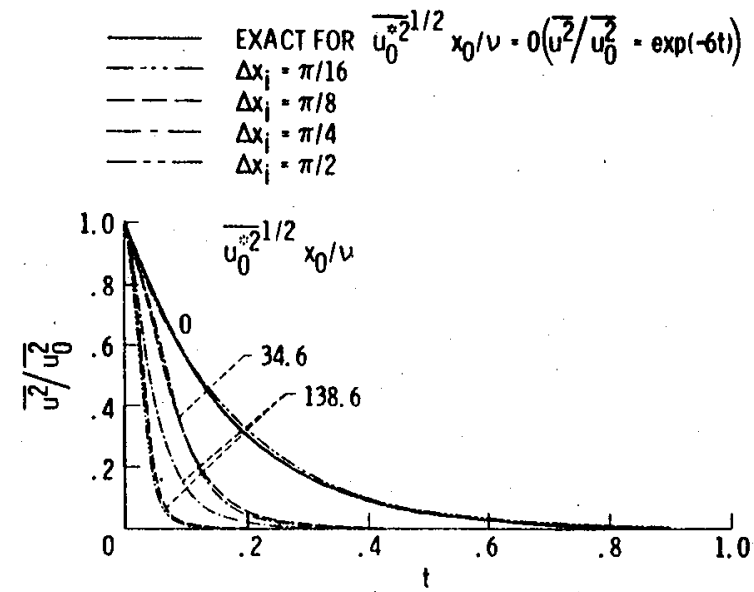

Fig. 5 Effect of numerical mesh size on evolution of $\overline{u^{2}}$ at low and moderate Reynolds numbers.

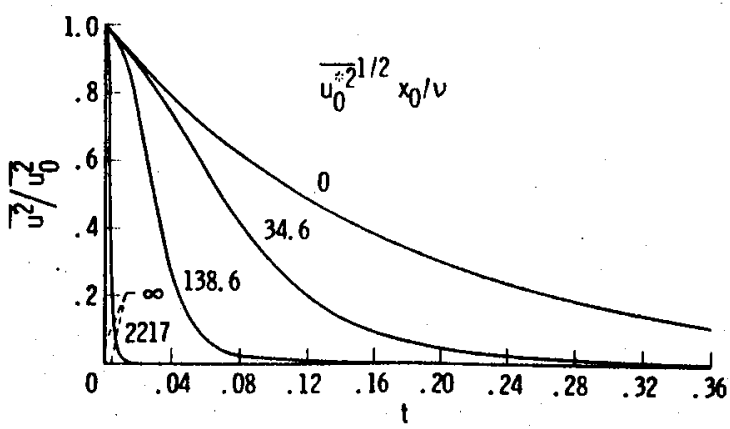

Fig. 6 Calculated evolution of mean-square velocity fluctuations (normalized by initial value) for various initial Reynolds numbers. 


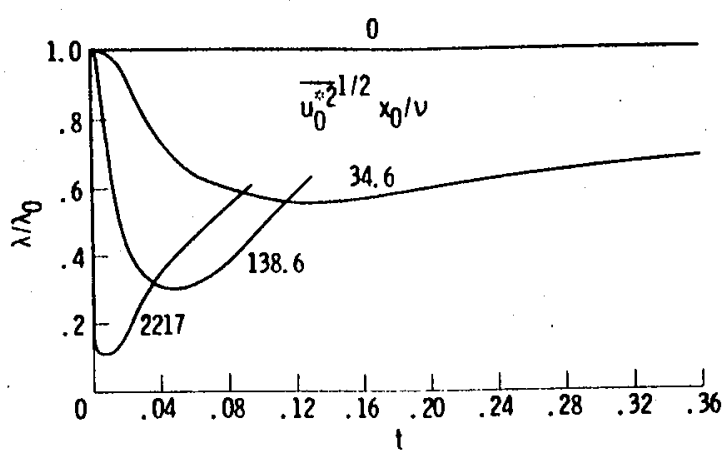

Fig. 7 Calculaled evolution of microscale of velocity fluctuations (normalized by initial value) for various initial Reynolds numbers.

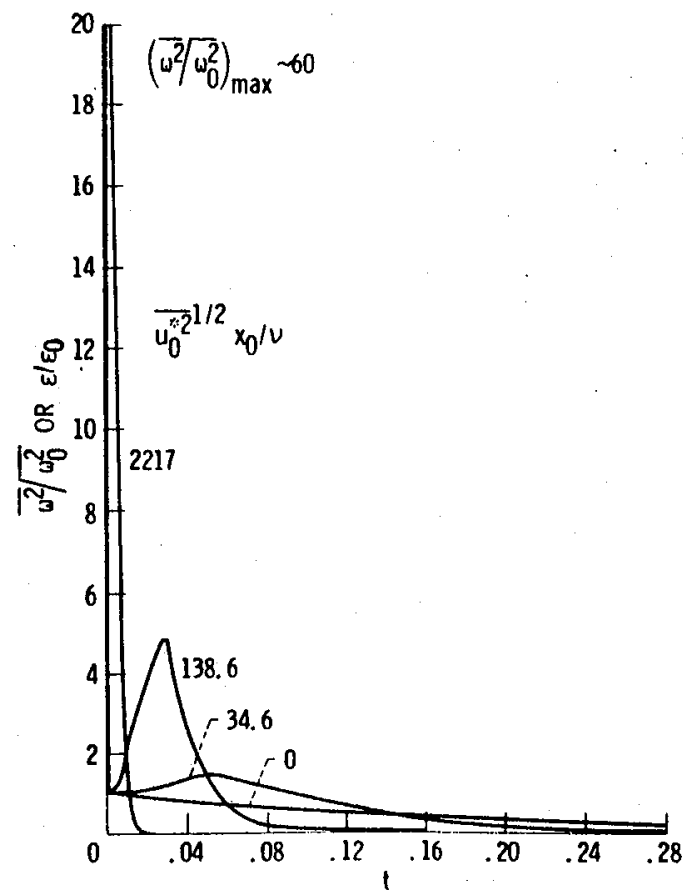

Fig. 8 Calculated development of mean-square vorticity fluctuations $\overrightarrow{w^{2}}$ or dissipation $\varepsilon$ (normalized by initial valuel for various inital Reynolds numbers. 


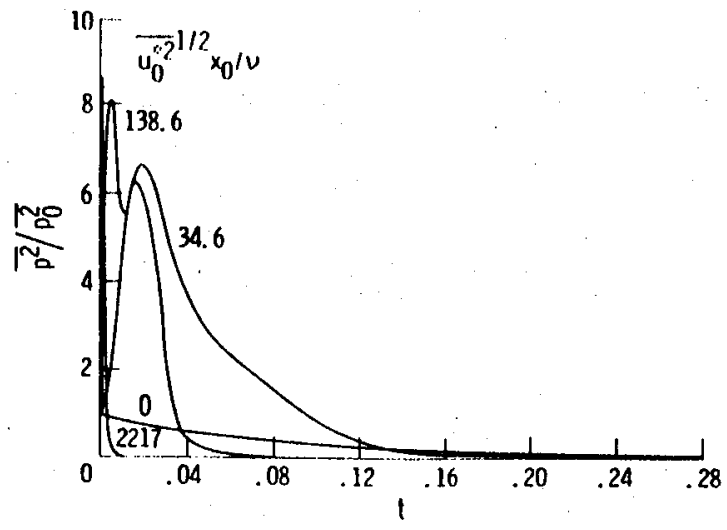

Fig. 9 Calculated evolution of mean-square pressure fluctuation (normalized by initial value) for various initial Reynolds numbers.

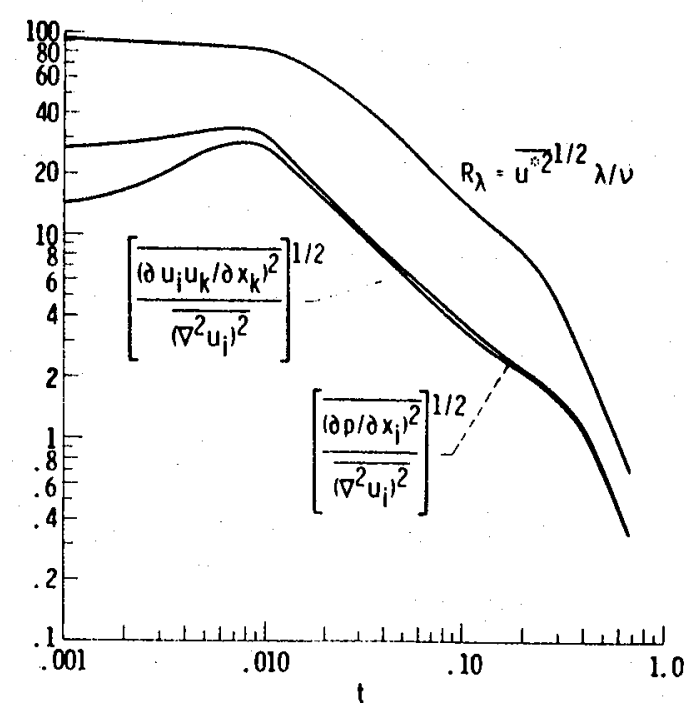

Fig. 10 Three measures of relative importance of inertial and viscous effects versus dimensionless time. $u_{0}^{x_{2}} 1 / 2 \quad x_{0} / v=69.3 . \quad i=1,2$, or 3 . 


\begin{tabular}{|c|c|c|c|c|}
\hline $\begin{array}{l}\text { 1. Report No. } \\
\text { NASA TM-81621 }\end{array}$ & \multicolumn{2}{|c|}{ 2. Government Accession No. } & \multicolumn{2}{|c|}{ 3. Recipient's Catalog No. } \\
\hline \multicolumn{3}{|l|}{ 4. Title and Subtitle } & \multicolumn{2}{|l|}{ 5. Report Date } \\
\hline \multicolumn{3}{|c|}{ TURBULENT SOLUTION OF THE NAVIER-STOKES EQUATIONS } & \multicolumn{2}{|c|}{ 6. Performing Organization Code } \\
\hline \multicolumn{3}{|l|}{$\begin{array}{l}\text { 7. Author(s) } \\
\text { R. G. Deissler }\end{array}$} & \multicolumn{2}{|c|}{$\begin{array}{l}\text { 8. Performing Organization Report No. } \\
\text { E-631 }\end{array}$} \\
\hline \multirow{2}{*}{\multicolumn{3}{|c|}{$\begin{array}{l}\text { 9. Performing Organization Name and Address } \\
\text { National Aeronautics and Space Administration } \\
\text { Lewis Research Center } \\
\text { Cleveland, Ohio } 44135\end{array}$}} & \multicolumn{2}{|l|}{ 10. Work Unit No. } \\
\hline & & & \multicolumn{2}{|c|}{ 11. Contract or Grant No. } \\
\hline \multirow{2}{*}{\multicolumn{3}{|c|}{$\begin{array}{l}\text { 12. Sponsoring Agency Name and Address } \\
\text { National Aeronautics and Space Administration } \\
\text { Washington, D.C. } 20546\end{array}$}} & \multicolumn{2}{|c|}{$\begin{array}{l}\text { 13. Type of Report and Period Covered } \\
\text { Technical Memorandum }\end{array}$} \\
\hline & & & \multicolumn{2}{|c|}{ 14. Sponsoring Agency Code } \\
\hline \multicolumn{5}{|c|}{$\begin{array}{l}\text { 15. Supplementary Notes } \\
\text { Prepared for the Thirty-third Annual Meeting of the Division of Fluid Dynamics of the American } \\
\text { Physical Society, Ithaca, New York, November } 23-25,1980 \text {. }\end{array}$} \\
\hline \multicolumn{5}{|c|}{$\begin{array}{l}\text { 16. Abstract } \\
\text { To study the nonlinear physics of incompressible turbulent flow, the unaveraged Navier-Stokes } \\
\text { equations are solved numerically. Initial three-dimensional cosine velocity fluctuations and } \\
\text { periodic boundary conditions are used. No mean gradients are present. The three components } \\
\text { of the mean-square velocity fluctuations are equal for the initial conditions chosen. The result- } \\
\text { ing solution shows characteristics of turbulence, such as the nonlinear excitation of small-scale } \\
\text { fluctuations. For the higher Reynolds numbers the initially nonrandom flow develops into an } \\
\text { apparently random turbulence. }\end{array}$} \\
\hline $\begin{array}{l}\text { 17. Key Words (Suggested by Auth } \\
\text { Turbulence; Fluid dy } \\
\text { equations; Numerical } \\
\text { Strange attractors }\end{array}$ & $\begin{array}{l}\text { Javier-Stokes } \\
\text { Randomization; }\end{array}$ & $\begin{array}{l}\text { 18. Distribution Stateme } \\
\text { Unclassified - } \\
\text { STAR Categor }\end{array}$ & unlimited & \\
\hline $\begin{array}{l}\text { 19. Security Classif. (of this report) } \\
\text { Unclassified }\end{array}$ & $\begin{array}{l}\text { 20. Security Classif. lo } \\
\text { Uncl: }\end{array}$ & $\begin{array}{l}\text { this page) } \\
\text { Issified }\end{array}$ & 21. No. of Pages & 22. Price \\
\hline
\end{tabular}

* For sale by the National Technical Information Service, Springfield. Virginia 22161 
National-Aerronautics and Space Administration

Washington, D.C.

20546

Official Business

Penalty for Private Use, $\$ 300$

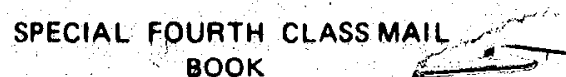

BOOK ostage and $F$ National Aero Space Administration NASA-451

4513 